\title{
Polymorphisms of DNA Repair Pathway Genes and Cigarette Smoking in Relation to Susceptibility to Large Artery Atherosclerotic Stroke among Ethnic Chinese in Taiwan
}

\author{
Hann-Yeh Shyu ${ }^{1,2}$, Jia-Ching Shieh ${ }^{3}$, Ji-Ho Lin ${ }^{4}$, Hsiao-Wei Wang ${ }^{5,6}$ and Chun-Wen Cheng ${ }^{5,6}$ \\ ${ }^{1}$ Section of Neurology, Department of Internal Medicine, Armed Forces Taoyuan General Hospital, Taoyuan, Taiwan \\ ${ }^{2}$ Institute of Biology and Anatomy, National Defense Medical Center, Taipei, Taiwan \\ ${ }^{3}$ Department of Biomedical Sciences, Chung Shan Medical University, Taichung, Taiwan \\ ${ }^{4}$ Department of Neurology, Taipei General Hospital, Department of Health, Executive Yuan, Taiwan \\ ${ }^{5}$ Clinical Laboratory, Chung Shan Medical University Hospital, Taichung, Taiwan \\ ${ }^{6}$ Institute of Biochemistry and Biotechnology, Chung Shan Medical University, Taichung, Taiwan
}

\begin{abstract}
Aim: Cigarette-smoking induced oxidative DNA damage to endothelial cells has been reported to play an etiological role in atherosclerosis development. Individual vulnerability to oxidative stress through smoking exposure and the ability to repair DNA damage, which plays a critical role in modifying the risk susceptibility of large artery atherosclerotic (LAA) stroke, is hypothesized. Thus, we examined the effect of genetic polymorphisms of DNA repair pathway genes and cigarette smoking in relation to risk susceptibility of LAA stroke.

Methods: We enrolled 116 LAA stroke patients and 315 healthy controls from the Armed Forces Taoyuan General Hospital, Taoyuan, Taiwan. Genotyping of polymorphisms of the OGG1 (Ser326Cys), XRCC1 (Arg399Gln), ERCC2 (Lys751Gln), and ERCC5 (Asp1104His) genes was performed and used to evaluate LAA stroke susceptibility.

Results: Of those non-synonymous polymorphisms, the ERCC2 Lys751Gln variant was found to be associated with LAA stroke risk (OR: 1.69, 95\%CI: 1.02-2.86), and this association was more pronounced in smokers, manifesting a 2.73 -fold increased risk of LAA stroke $(p=0.027)$. A joint effect on risk elevation of LAA stroke was seen in those patients with $O G G 1$ and ERCC2 polymorphisms (OR: 2.75, 95\%CI: 1.26-6.00). Moreover, among smokers carrying the OGG1 Ser326Cys polymorphism, there was a tendency toward an increased risk of LAA stroke in those patients who had a greater number of high-risk genotypes of XRCC1, ERCC2, and ERCC5 polymorphisms ( $p_{\text {trend }}=0.010$ ). Conclusion: The susceptible polymorphisms of DNA repair pathway genes may have a modifying effect on the elevated risk of LAA stroke in smokers among ethnic Chinese in Taiwan.
\end{abstract}

J Atheroscler Thromb, 2012; 19:316-325.

Key words; Large artery atherosclerotic stroke, DNA repair genes, Polymorphism, Smoking, Susceptibility

Abbreviations; LAA: Large artery atherosclerotic, BER: Base excision repair, NER: Nucleotide excision repair, ROS: Reactive oxygen species, SNP: Single nucleotide polymorphism, OGG1: 8-oxoguanine glycosylase 1, XRCC1: X-ray repair cross complementing group 1, ERCC2: Excision repair cross complementation group 2, ERCC5: Excision repair cross complementation group 5

Address for correspondence: Chun-Wen Cheng, Ph.D., Institute of Biochemistry and Biotechnology, Chung Shan Medical University, No. 110, Sec. 1, Chien-Kuo N. Road, Taichung 40201, Taiwan

E-mail: cwcheng@csmu.edu.tw

Received: June 29, 2011

Accepted for publication: September 22, 2011

\section{Introduction}

Stroke remains the major leading cause of death worldwide, including in the ethnic Taiwanese population. Stroke is believed to be a polygenic multifactori- 
al model of etiology caused by the sum of genetic and environmental factors ${ }^{1,2)}$. Cigarette smoking has been considered as an important risk factor for atherosclerotic vascular diseases, including coronary artery disease $(\mathrm{CAD})$ and cerebrovascular disease $(\mathrm{CVD})^{3,4)}$. Although the mechanism underlying cigarette smoke-induced cerebrovascular injury regarding to stroke remains unclear, cigarette constituents are known to activate reactive oxygen species (ROS) on DNA damage within the vascular wall, leading to premature vascular aging and endothelial dysfunction ${ }^{3,5,6)}$.

A direct causality in which exposure to cigarette smoke results in elevated levels of DNA aberrations in various human cell types, including fibroblasts, lymphocytes and endothelial cells has been shown in earlier reports ${ }^{7,8)}$. Recently, accumulating evidence from cohort studies revealed that the increased risk of stroke is generally associated with smoking ${ }^{9,10)}$. The smoking-related oxidized bases, such as 7,8-dihydro-8-oxoguanine (8-oxo-G) and 8-hydroxy-2'-deoxyguanosine (8-OHdG), are extensively studied DNA destroyers that result in mutagenic guanine base lesions through the action of ROS and lead ultimately to cell tumorigenesis ${ }^{11,12)}$. In addition, the presence of cigarette smoking-related polycyclic aromatic hydrocarbon (PAH)-DNA adducts has been detected in human mammary artery specimens from smokers ${ }^{13)}$, indicating that cigarette smoke can induce chromosomal aberrations in the cardiovascular system. As the cumulative oxidative stress caused by smoking is capable of inducing DNA damage that may correlate with the vascular aging process ${ }^{3,14)}$, an underlying mechanism is thus hypothesized in which individual vulnerability to oxidative stress through smoking exposure and polymorphisms of DNA repair genes, with accumulated oxidative DNA damage together with deficits in DNA repair enzymes, plays a critical role in modifying the risk susceptibility to stroke among smokers.

Base excision repair (BER) and nucleotide excision repair (NER) pathways are two mainly DNA damage repair systems, which are involved in eliminating and repairing damaged DNA caused by methylating chemicals and ROS. 8-Oxoguanine glycosylase 1 (OGG1) enzyme is involved in the excision of 8-oxoguanine, a highly mutagenic compound that can form mismatched base-pairs in DNA. A transversion mutation of the OGG1 gene with Ser326Cys (rs1052133) polymorphism has been known to encode a $N$-glycosylase/DNA lyase with decreased enzyme activity that leads to 8-oxoguanine accumulation ${ }^{12,15)}$. X-ray repair cross complementing group 1 (XRCC1) acts as a scaffolding protein that interacts with poly (ADP-ribose) polymerase, DNA polymerase- $\beta$ and DNA ligase III in BER ${ }^{16)}$. Excision repair cross complementation group 2 (ERCC2, also known as XPD) encodes an ATP-dependent DNA helicase that interacts with transcription factor BTF2/ TFIIH $^{17)}$ and ERCC5 (known as XPG) genes takes part in incision steps to respond to a wide range of structurally bulky lesions in NER ${ }^{18)}$. To date, known polymorphisms in OGG1 (Ser326Cys), XRCC1 (Arg 399Gln), ERCC2 (Lys751Gln), and ERCC5 (Asp1104 $H i s)$ have been reported to alter the ability of encoded enzymes to repair DNA damage, and those variant alleles have been found in individuals predisposed to increased risk of many kind of cancers and autoimmune disorders ${ }^{19-22)}$.

Although association studies of the polymorphisms of candidate genes contributing to individual susceptibility to stroke disease have been performed ${ }^{23}{ }^{24)}$, knowledge of variations in DNA repair enzymes regarding to stroke development is relatively limited. Moreover, extended information remains unclear on DNA repair gene polymorphisms linked to smoking regarding the risk modification of stroke. In this study, we elucidated whether inter-individual differences in these four non-synonymous SNPs in BER (OGG1 Ser326Cys and XRCC1 Arg399Gln) and NER (ERCC2 Lys751Gln and ERCC5 Asp1104His) pathway genes might predispose carriers to an increase risk of large artery atherosclerotic (LAA) stroke, especially in the subgroup of smokers among ethnic Chinese in Taiwan.

\section{Methods}

\section{Study Population}

In this hospital-based case-control study, demographic data were collected from 116 consecutive patients with LAA stroke disease and 315 age- and gender-matched healthy controls. All participants were included upon receipt of their informed consent according to a protocol approved by the Ethics Committee of Institutional Review Board of the Armed Forces Taoyuan General Hospital, Taoyuan, Taiwan. Ischemic stroke (IS) was defined according to the World Health Organization definition as "rapidly developing clinical signs of focal (or global) disturbance of cerebral function lasting more than 24 hours with no apparent cause other than of vascular origin". Any patients with atypical symptoms, including transient ischemic attacks, intracranial hemorrhage, postseizure palsy, brain trauma, or brain tumors, were excluded from the study. All IS patients underwent at least one brain imaging (CT and/or MRI) study. Based on the clinical features examined by brain imaging, cardiac imaging (echocardiography), ultrasonography of ex- 
tracranial and intracranial arteries, and angiography (MRA or conventional angiography), these IS patients were subclassified according to the TOAST classification system ${ }^{25}$ 26). The healthy control group consisted of 315 individuals recruited from the same hospital in the health examination clinic during the same study period, and each of these controls was chosen on the basis that they showed no evidence of any cerebrovascular disorders. No significant differences were observed in socioeconomic status between those who were and were not included.

\section{Data Collection}

An experienced research nurse was assigned to administer a systemic questionnaire to both the case and control groups. The information collected included demographic characteristics (ethnic background, residence area, and educational level), medical history (age at diagnosis and family history of stroke, hypertension, diabetes mellitus, and ischemic heart disease), a history of current alcoholism (referred to as alcohol abuse, which was described in the Diagnostic and Statistical Manual of Mental Disorders IV) [American Psychiatric Association, Diagnostic and Statistical Manual of Mental Disorders (4th ed.), American Psychiatric Association, Washington, DC (1994)], a habit of daily cigarette smoking (in this study, all the smokers had consumed more than 10 cigarettes daily for at least 10 years) as well as parameters of hypercholesterolemia (total cholesterol $\geq 200 \mathrm{mg} / \mathrm{dl}$ and/or low-density lipoprotein cholesterol $\geq 130 \mathrm{mg} / \mathrm{dl}$ ) were recorded.

\section{Genotype Analysis}

A sample of $10 \mathrm{ml}$ peripheral blood, collected in acetate-citrate dextrose, was obtained from a fasting venous blood sample that was drawn from each patient and the control subject. Genomic DNA was extracted from peripheral blood lymphocytes by a QIAamp DNA blood mini kit (QIAGEN Inc., Chatsworth, CA, USA) following the manufacturer's protocol and was then stored at $-20^{\circ} \mathrm{C}$ for subsequent genotype determination. Those non-synonymous variants of interest were chosen for genotyping based on the following criteria: (i) single nucleotide polymorphism (SNP) resulted in an amino acid substitution for biological considerations; and (ii) minor allelic frequency of the selected allele was observed to be higher than five percent due to consideration of statistical power; and (iii) information on gene single nucleotide polymorphisms (SNPs) in DNA repair pathways were reported among ethnic Chinese population arranged in the HapMap database (http://www.hapmap.org/). Genotyping de- termination of gene variants was carried out using TaqMan Assays-by-Design (Applied Biosystems, Foster City, CA). SNP information was obtained from the public SNP database (http://www.ncbi.nlm.nih. gov/SNP/index.html, National Institute of Health, USA). DNA sequences of probes and primers for the genotyping assays were chosen using the TaqMan Assays-by-Design (Applied Biosystems), including the Arg399Gln (G-to- $A$ transition, rs25487) in XRCC1, Ser326Cys ( $G$-to- $C$ transition, rs1052133) in $O G G 1$, Lys751Gln (A-to-C transition, rs13181) in ERCC2, and Asp1104His (G-to-C transition, rs17655) in the ERCC5 gene. Genotype analyses of these variant alleles were performed using the TaqMan assay and PCR amplification. PCR reactions were performed on a GeneAmp PCR System (ABI Prism 7000 Sequence Detection System; Applied Biosystems), using $20 \mu 1$ containing a $200 \mathrm{nM}$ probe, $900 \mathrm{nM}$ primer set, 1X TaqMan Universal PCR Master Mix (Applied Biosystems), and 50 ng DNA templates. For quality assurance, independent positive and negative control samples were included and analyzed in each 96-well plate in each batch. To ensure that the observed polymorphisms were specific and not the result of experimental variations, all uncertain genotypic results were subjected to a repeating assay for confirmation.

\section{Statistical Analyses}

Means and standard deviations or frequencies and percents were calculated for continuous and categorical variables, respectively. The allelic and genotypic frequencies of each polymorphism for these four genes were compared between cases and control subjects using the chi-square test or Fisher's exact test as appropriate. The adjusted odds ratio (OR) and the corresponding $95 \%$ confidence interval $(95 \% \mathrm{CI})$ for the association was estimated considering known risk factors for IS in multivariate logistic regression analysis. Exposure to cigarette smoking was hypothesized to contribute to an elevated risk of LAA stroke. In addition, individuals who carried high-risk alleles, such as the variant allele of XRCC1 Gln399 and OGG1 Cys326 of the BER pathway, as well as ERCC2 Gln751 and ERCC5 1104His of the NER pathway, mimicked functional deficits in cells with lower efficacy in response to DNA damage repair in blood endothelial vessel tissues, were thus considered to be higher risk genotypes of LAA stroke development. Further, to examine the joint effect of these variants, we assumed that each variant genotype of the SNPs contributed equally to the increased risk of LAA stroke, and individuals who carried the same number of putative highrisk genotypes would have the same elevated risk sus- 
Table 1. Demographic data for LAA stroke patients and healthy controls

\begin{tabular}{|c|c|c|c|}
\hline Variable $^{\S}$ & $\begin{array}{c}\text { Cases }(N=116) \\
\text { No }(\%)\end{array}$ & $\begin{array}{c}\text { Controls }(N=315) \\
\text { No }(\%)\end{array}$ & OR $(95 \%$ CI $)$ \\
\hline \multicolumn{4}{|l|}{ Age } \\
\hline Mean \pm SD & $72.5 \pm 11.0$ & $71.7 \pm 6.6$ & $1.37(0.89-2.16)$ \\
\hline \multicolumn{4}{|l|}{ Sex } \\
\hline Men & $82(70.7)$ & $171(54.3)$ & $1.86(0.93-3.29)$ \\
\hline Women & $34(29.3)$ & $144(45.7)$ & \\
\hline Diabetes mellitus & $35(29.9)$ & $47(14.9)$ & $2.46(1.41-4.20)^{* *}$ \\
\hline Hypertension & $78(66.7)$ & $139(44.1)$ & $2.60(1.62-4.17)^{* *}$ \\
\hline Hypercholesterolemia & $51(43.6)$ & $108(34.3)$ & $1.50(0.96-2.38)$ \\
\hline Alcoholism & $20(17.1)$ & $41(13.0)$ & $1.39(0.75-2.54)$ \\
\hline Smoking & $42(36.2)$ & $77(24.4)$ & $1.75(1.07-2.84)^{*}$ \\
\hline
\end{tabular}

ceptibility to LAA stroke. Therefore, the effects of the multiple interactions of genes on LAA stroke risk were evaluated in subjects carrying a combination of susceptible genotypes. All statistical analyses were performed by an experienced statistician. All statistical tests were performed with SPSS version 17.0 based on a 2 -tailed probability and $p<0.05$ was considered significant.

\section{Results}

Distributions of the demographics of the cases and healthy controls are summarized in Table 1 . The patients and healthy controls had a similar average age. Among 116 LAA stroke patients diagnosed with large artery atherosclerosis (mean age: $72.5 \pm 11.0$ years), 82 were men (mean age: $72.4 \pm 11.3$ years) and 34 were women $(72.6 \pm 10.6$ years), respectively. A total of 315 healthy controls (mean age: $71.7 \pm 6.6$ years) were enrolled, including 171 men (mean age: $72.2 \pm 6.9$ years) and 144 women (mean age: $71.4 \pm 5.9$ years). Compared to healthy control subjects, an increased LAA stroke risk was found to be associated with known risk factors, including diabetes mellitus (OR: 2.46, 95\%CI : 1.41-4.20), hypertension (OR: 2.60, 95\% CI: 1.62-4.17), and cigarette smoking (OR: 1.75, 95\%CI: 1.07-2.84) using multiple logistic re- gression analysis (Table $\mathbf{1}$ ).

Frequencies of genotype polymorphisms for XRCC1, OGG1, ERCC2 and ERCC5 genes between cases and control subjects are shown in Table 2. Distributions of the observed allelic and genotypic frequencies of these polymorphisms were in HardyWeinberg equilibrium in the controls. Due to the fact that the variant genotypes of these polymorphisms were reported to result in lower enzymatic activities and that a small percentage of subjects had the homozygous variant genotype of these genes in our cohort, individuals carrying heterozygous and homozygous variant genotypes for these genes were grouped and compared to the homozygous wild-type genotype, and such grouping strategy has also been widely used in previous molecular epidemiological studies $\left.{ }^{15}, 16,27,28\right)$. Among 431 subjects collected from the Armed Forces Taoyuan General Hospital, Taoyuan, Taiwan, we found no significant differences in allelic and genotypic frequencies for these four genotypic polymorphisms regarding age at LAA stroke onset ( $\leq 70$ vs. $>70$ years) or gender $(p>0.05)$. Comparing cases and control subjects, genotype frequencies of either heterozygous or homozygous variants of XRCC1, OGG1, and ERCC5 were found higher in the cases; however, the variant genotypes of these genes showed insignificantly increased LAA stroke risk after they were adjusted 
Table 2. Genotype frequencies of polymorphisms of DNA repair genes among cases and controls and odds ratio in relation to risk of LAA stroke

\begin{tabular}{|c|c|c|c|c|c|}
\hline Gene and $\mathrm{SNP}^{\S}$ & No. cases $(\%)$ & No. controls (\%) & $\mathrm{aOR}(95 \% \mathrm{CI})^{\dagger}$ & aOR for trend ${ }^{\#}$ & $p$ for permutation \\
\hline \multicolumn{6}{|c|}{ XRCC1 Arg399Gln (rs25487; G>A) } \\
\hline Arg/Arg & $55(47.4)$ & $170(54.0)$ & 1.00 (Ref) & $1.19(0.84-1.69)$ & 0.238 \\
\hline $\mathrm{Arg} / G \ln$ & $49(42.2)$ & $119(37.8)$ & $1.37(0.87-2.17)$ & & \\
\hline$G \ln / G \ln$ & $12(10.4)$ & $26(8.2)$ & $1.43(0.69-3.05)$ & & \\
\hline \multicolumn{6}{|c|}{ OGG1 Ser326Cys (rs1052133C>G) } \\
\hline Ser/Ser & $25(21.5)$ & $86(27.3)$ & 1.00 (Ref) & $1.13(0.83-1.54)$ & 0.265 \\
\hline Ser/Cys & $51(44.0)$ & $141(44.8)$ & $1.27(0.73-2.23)$ & & \\
\hline Cys/Cys & $40(34.5)$ & $88(27.9)$ & $1.69(0.93-3.04)$ & & \\
\hline \multicolumn{6}{|c|}{ ERCC2 Lys751Gln (rs13181; $A>C)$} \\
\hline Lys/Lys & $87(75.0)$ & $262(83.2)$ & 1.00 (Ref) & $1.56(0.97-2.51)$ & 0.062 \\
\hline Lys/Gln & $24(20.7)$ & $48(15.2)$ & $1.47(0.85-2.54)$ & & \\
\hline$G \ln / G \ln$ & $5(4.3)$ & $5(1.6)$ & $3.03(0.87-9.32)$ & & \\
\hline \multicolumn{6}{|c|}{ ERCC5 Asp1104His (rs17655; G>C) } \\
\hline Asp/Asp & $29(25.0)$ & $108(34.3)$ & 1.00 (Ref) & $1.23(0.91-1.68)$ & 0.081 \\
\hline Asp/His & $61(52.6)$ & $142(45.1)$ & $1.61(0.95-2.67)$ & & \\
\hline His/His & $26(22.4)$ & 65 (20.6) & $1.50(0.81-2.78)$ & & \\
\hline
\end{tabular}

§ The 'rs' number shown is the National Center for Biotechnology Information dbSNP cluster ID for each SNP.

$\dagger \mathrm{aOR}$ and corresponding $95 \%$ confidence interval $(95 \% \mathrm{CI})$ were calculated by logistic regression, in which a set of dummy variables represented different genotypes of polymorphisms of DNA repair genes. In addition, these statistical analyses were performed considering age, sex, alcoholic, cigarette smoking, diabetes mellitus, hypertension, and hypercholesterolemia in the regression model.

\# The aOR for trend indicates the odds ratio associated with subjects carrying one additional variant allele of individual SNPs, and it was estimated in the logistic regression model, in which the genotype was treated as a continuous variable.

Abbreviations: Ref, reference group.

in the multiple logistic regression model (Table 2). The results of genotypic analysis of ERCC2 Lys $751 \mathrm{Gln}$ was important, as it showed that subjects carrying the homozygous variant genotype had an increased LAA stroke risk compared to carriers with the homozygous wild-type genotype, and that carrying one additional risk allele was associated with a 1.56-fold increased OR in LAA stroke risk (95\%CI: 0.97-2.51). The possibility of a false positive due to multiple testing is less likely, because our permutation test based on 10,000 random permutations showed that this association was borderline significant $(p=0.062)$ (Table 2). Additionally, considering subjects who carried the homozygous ERCC2 Lys751 genotype as a reference, we found an OR of 1.69-fold increase (95\%CI: 1.02-2.86, $p=0.047$ ) for combined heterozygous and homozygous Lys 751 Gln variants in LAA stroke risk.

The modified effect of the variant genotypes of individual genes on risk association between cigarette smoking and LAA stroke are shown in Table 3. Smoking habits, as described in Methods, were classified into two groups. Using Lys751Lys as a reference, a greater proportion of the ERCC2 codon $751 G \ln$ variant (Lys751Gln and Gln751Gln) was seen in the cases, conferring a significantly increased 2.73-fold risk in that subset of smokers (95\%CI: 1.12-6.65, $p=0.027$ ) in the multivariate logistic regression model. When compared to smokers carried the respective wild-type genotypes, a higher frequency of the combined heterozygous and homozygous genotypes of the Gln399 allele for XRCC1, Cys326 allele for the $O G G 1$, and His 1104 allele for ERCC5 was found in LAA stroke patients than in control subjects; however, the modified effect of these polymorphisms on risk association between smoking and LAA stroke was insignificant (Table 3). In addition, we explored the joint effect of these DNA repair genes by determining the association between gene-to-gene interactions and susceptibility to LAA stroke. Using a dummy variable coding scheme and the $\beta$ estimate from the logistic regression model $^{29)}$, subjects with both polymorphisms of $O G G 1$ Ser326Cys and ERCC2 Lys751Gln have a 2.75-fold increased OR risk compared to those with respective wild-type genotypes $(p=0.011)($ Table 4$)$. 
Table 3. Joint effects of genotypic polymorphisms in individual gene and smoking status on risk susceptibility of LAA stroke

\begin{tabular}{|c|c|c|c|c|c|c|}
\hline \multirow{2}{*}{ Genotype } & \multicolumn{3}{|c|}{ Non-smoker } & \multicolumn{3}{|c|}{ Smoker } \\
\hline & Case/Control & OR $(95 \% \mathrm{CI})^{\S}$ & $p$ value & Case/Control & OR $(95 \% \mathrm{CI})^{\S}$ & $p$ value \\
\hline \multicolumn{7}{|l|}{ XRCC1 Arg399Gln } \\
\hline Arg/Arg & $34 / 121$ & $1.00(\operatorname{Ref})^{\mathrm{b}}$ & 0.396 & $21 / 49$ & 1.00 (Ref) & 0.157 \\
\hline Arg/Gln, Gln/Gln & $40 / 117$ & $1.26(0.74-2.14)$ & & $21 / 28$ & $1.73(0.81-3.73)$ & \\
\hline \multicolumn{7}{|l|}{ OGG1 Ser326Cys } \\
\hline Ser/Ser & $14 / 57$ & 1.00 (Ref) & 0.417 & $11 / 29$ & 1.00 (Ref) & 0.189 \\
\hline Ser/Cys, Cys/Cys & $60 / 181$ & $1.32(0.68-2.55)$ & & $31 / 48$ & $1.75(0.76-4.03)$ & \\
\hline \multicolumn{7}{|l|}{ ERCC2 Lys751Gln } \\
\hline Lys/Lys & $59 / 197$ & 1.00 (Ref) & 0.445 & $28 / 65$ & 1.00 (Ref) & 0.027 \\
\hline Lys/Gln, Gln/Gln & $15 / 41$ & $1.30(0.67-2.54)$ & & $14 / 12$ & $2.73(1.12-6.65)^{*}$ & \\
\hline \multicolumn{7}{|l|}{ ERCC5 Asp1104His } \\
\hline$A s p / A s p$ & $19 / 80$ & 1.00 (Ref) & 0.325 & $10 / 28$ & 1.00 (Ref) & 0.168 \\
\hline Asp/His, His/His & $55 / 158$ & $1.35(0.74-2.45)$ & & $32 / 49$ & $1.82(0.78-4.35)$ & \\
\hline
\end{tabular}

${ }^{\S}$ Multivariate analysis of OR and $95 \% \mathrm{CI}$, and $p$ value was assessed in a logistic regression model after adjustment for age, sex, diabetes mellitus, hypertension, and hypercholesterolemia.

Table 4. Interaction between polymorphisms of the OGG1 Ser326Cys and different DNA repair genes in LAA stroke risk

\begin{tabular}{|c|c|c|c|c|c|}
\hline Gene/polymorphisms & & $\begin{array}{l}\text { Cases } \\
n(\%)\end{array}$ & $\begin{array}{c}\text { Controls } \\
n(\%)\end{array}$ & OR $(95 \% \mathrm{CI})^{\S}$ & $p$ value \\
\hline$O G G 1$ & $X R C C 1$ & & & & \\
\hline Ser/Ser & Arg/Arg & $12(10.3)$ & $51(16.2)$ & 1.00 (Ref) & \\
\hline Ser/Cys, Cys/Cys & Arg/Arg & $43(37.1)$ & $119(37.8)$ & $1.58(0.76-3.27)$ & 0.217 \\
\hline Ser $/$ Ser & $A r g / G \ln , G \ln / G \ln$ & $13(11.2)$ & $35(11.1)$ & $1.62(0.65-3.98)$ & 0.301 \\
\hline Ser/Cys, Cys/Cys & Arg/Gln, Gln/Gln & $48(41.4)$ & $110(34.9)$ & $1.99(0.96-4.11)$ & 0.062 \\
\hline$O G G 1$ & ERCC2 & & & & \\
\hline Ser/Ser & Lys/Lys & $17(14.7)$ & $66(21.0)$ & 1.00 (Ref) & \\
\hline Ser/Cys, Cys/Cys & Lys/Lys & $70(60.3)$ & $196(62.2)$ & $1.42(0.77-2.60)$ & 0.258 \\
\hline $\mathrm{Ser} / \mathrm{Ser}$ & $L y s / G \ln , G \ln / G \ln$ & $8(6.9)$ & $20(6.3)$ & $1.52(0.57-4.09)$ & 0.406 \\
\hline Ser/Cys, Cys/Cys & Lys/Gln, Gln/Gln & $21(18.1)$ & $33(10.5)$ & $2.75(1.26-6.00)$ & 0.011 \\
\hline$O G G 1$ & ERCC5 & & & & \\
\hline Ser/Ser & Asp/Asp & $5(4.3)$ & $30(9.5)$ & 1.00 (Ref) & \\
\hline Ser/Cys, Cys/Cys & Asp/Asp & $24(20.7)$ & $78(24.8)$ & $1.77(0.61-5.12)$ & 0.290 \\
\hline $\mathrm{Ser} / \mathrm{Ser}$ & Asp/His, His/His & $20(17.2)$ & $56(17.8)$ & $1.85(0.62-5.49)$ & 0.267 \\
\hline Ser/Cys, Cys/Cys & Asp/His, His/His & $67(57.8)$ & $151(47.9)$ & $2.47(0.91-6.72)$ & 0.075 \\
\hline
\end{tabular}

$\S$ Multivariate analysis of the adjusted odds ratio and 95\% confidence intervals were estimated in a logistic regression model after adjustment for age, sex, diabetes mellitus, hypertension, and hypercholesterolemia.

Considering an increased LAA stroke risk led by the combined effect of these four polymorphisms being the result of decreased availability of active DNA repair enzymes regarding DNA damage, we expected that subjects with a greater number of high-risk genotypes would be even more susceptible to LAA stroke development if they had been exposed to smoking en- vironments. Based on the stratification of $O G G 1$ Cys326 polymorphism, we then classified our subjects into two groups according to XRCC1, ERCC2, and ERCC5 polymorphisms; those with no more than one high-risk genotype $(\leq 1)$ and those with at least two high-risk genotypes (2-3) in genes that participate in repairing DNA damage, and analyzed LAA stroke risk, 
Table 5. Combined effects of DNA repair pathway genes on LAA stroke risk stratified by cigarette smoking

\begin{tabular}{|c|c|c|c|c|c|c|c|}
\hline \multirow{2}{*}{ Genotype } & \multirow{2}{*}{$\begin{array}{l}\text { Number of high-risk } \\
\text { genotypes }\end{array}$} & \multicolumn{3}{|c|}{ Non-smoker } & \multicolumn{3}{|c|}{ Smoker } \\
\hline & & Case/Control & OR $(95 \% \mathrm{CI})^{\dagger}$ & $p$ value & Case/Control & OR $(95 \% \mathrm{CI})^{\dagger}$ & $p$ value \\
\hline$O G G 1$ & XRCC1/ERCC2/ERCC5 & & & & & & \\
\hline Ser/Ser & $\leq 1$ & $5 / 32$ & $1.00(\mathrm{Ref})^{\#}$ & & $5 / 18$ & $1.00(\text { Ref })^{\#}$ & \\
\hline Ser/Cys, Cys/Cys & $\leq 1$ & $32 / 106$ & $1.84(0.66-5.15)$ & 0.248 & $15 / 35$ & $1.56(0.49-4.96)$ & 0.460 \\
\hline Ser/Ser & $2-3$ & $9 / 25$ & $2.23(0.67-7.59)$ & 0.198 & $6 / 11$ & $1.98(0.48-7.99)$ & 0.346 \\
\hline Ser/Cys, Cys/Cys & $2-3$ & $28 / 75$ & $2.33(0.82-6.63)$ & 0.114 & $16 / 13$ & $4.43(1.29-15.19)$ & 0.018 \\
\hline & & & & $p_{\text {trend }}=0.138$ & & & $p_{\text {trend }}=0.01 \mathrm{C}$ \\
\hline
\end{tabular}

${ }^{\S}$ High-risk genotypes of DNA repair genes are identified as combined genotypes of heterozygous and homozygous variants of the polymorphisms of XRCC1 (Arg399Gln), ERCC2 (Lys751Gln), and ERCC5 (Asp1104His).

${ }^{\dagger}$ A multivariate logistic regression model was estimated after considering age, sex, diabetes mellitus, hypertension, and hypercholesterolemia.

\# Ref, reference group; risks were estimated using subjects carrying no more than one high-risk genotypes $(0-1)$ as the reference.

because such a definition would provide statistical power to address the question. As a result, when we considered those who carried the homozygous genotype of $O G G 1$ Ser326 and no more than one risk genotype of XRCC1, ERCC2, and ERCC5 polymorphisms as a reference, the combined effect of gene-to-gene interaction on increased LAA stroke risk was shown in smokers who carried Cys326 variants together with a greater number of high-risk genotypes of XRCC1, ERCC2, and ERCC5 (OR: 4.43, 95\%CI: 1.29-15.19, $p=0.018$ ) (Table 5). Moreover, using a dummy variable coding scheme and the $\beta$ estimate from the regression model ${ }^{30,31)}$, a tendency toward an increased LAA stroke risk was statistically significant in smokers who carried OGG1 Cys326 polymorphisms and greater numbers of high-risk genotypes $\left(p_{\text {trend }}=0.010\right)(\mathrm{Ta}-$ ble 5).

\section{Discussion}

In this study, we attempted to analyze the association between genotype polymorphisms of BER and NER pathway genes and susceptibility in patients with LAA stroke. Support for our hypothesis came from observations that the joint effect of $O G G 1$, XRCC1, ERCC2, and ERCC5 polymorphisms significantly increased LAA stroke risk, and this association between high-risk genotypes of multiple genes and LAA stroke development was more significant in smokers. In the BER pathway, the Ser326Cys variant in the $O G G 1$ gene was known to correlate with elevated levels of oxidized metabolites, such as 8-oxo-G and
$8-\mathrm{OHdG}$, in lung cancer among smokers ${ }^{32)}$. The presence of the Ser326Cys polymorphism was associated with cancers, diabetes mellitus, and neurodegenerative disorders in molecular epidemiological studies ${ }^{22,33-35)}$. In addition, it has been reported that subjects who carry the OGG1 Ser326Cys polymorphism are prone to multi-vessel disease in CAD among Chinese in Taiwan $^{36}$. In this study, the combined genotypes of heterozygous and homozygous OGG1 Cys326 variant showed insignificantly increased LAA stroke risk when compared to the homozygous Ser326 genotype. The power calculations in LAA stroke risk of this polymorphism are inconsistent. The major limitation in most molecular association studies is commonly ascribed to differential frequencies of unique SNPs among genetically susceptible individuals. Thus, an investigation of genetic susceptibility with a larger sample size is the best solution to resolve the current discrepancies regarding OGG1 Cys32 in LAA stroke. On the other hand, after the subgroup stratified by the Ser326Cys polymorphism for the OGG1 gene, the ERCC2 Lys $751 G l n$ variant genotype showed a significantly elevated risk in association with LAA stroke (Table 4). These findings suggest that OGG1 Ser326Cys, although not a genetic marker known to be independently associated with LAA stroke through the BER pathway, may critically and synergistically interact with individual genotypic polymorphisms of the NER pathway regarding increased LAA stroke risk. Moreover, the present case-control study is the first report of an association of DNA repair genes and LAA stroke risk in ethnic Chinese in Taiwan. It is important to 
pay attention to further genetic studies among other races, such as different Asian, Caucasian, and African populations, to clarify the role of the polymorphism in the $O G G 1$ in the investigation of stroke.

Inter-individual variations in decreasing NER capacities were seen in carriers of the ERCC2 Lys 751 Gln and ERCC5 Asp1104His polymorphisms and have been reported to correlate with susceptibility to can$\operatorname{cer}^{37,38)}$. In this study, we found that the homozygous ERCC2 Gln751 genotype is associated with an increased risk in LAA stroke, and this risk is up to 2.73-fold in smokers carrying the Gln 751 polymorphism of ERCC2 (Table 3). A growing list of evidence has shown that cigarette smoking can result in high levels of bulky DNA adducts in the rodent heart and aorta $^{39,40)}$. It has also been reported that involuntary exposure to smoking environments in those nonsmokers is significantly associated with coronary heart disease $^{41,42}$. In addition, the increased lung cancer incidence among smokers influenced by Gln751 polymorphism has been reported to be the result of accumulated levels of chromosomal aberrations and micronuclei in peripheral cells $\left.{ }^{43}, 44\right)$. Given that smokers with mutations in the genes in the NER pathway are defective in their ability to remove DNA cross-links, bulky polycyclic aromatic hydrocarbons (PAH)-DNA adduct, and oxidized base lesions, which may be harmful to endothelial and vascular smooth muscle cells, increased LAA stroke risk due to this polymorphism with relative lower enzyme activity to DNA repair is mechanistically reasonable. Hence, in epistatic analysis, compared to subjects with the ERCC2 Lys751 wild-type genotype, our data justify the role of the Gln751 polymorphism in smokers with greater susceptibility to LAA stroke.

Importantly, potentiated by multiple gene effects, cigarette smoking appears to be the predominant risk of LAA stroke; therefore, the assertion that the unique genotype polymorphisms for BER and NER pathways increase LAA stroke risk may be a result of the multiple gene make-up of the smokers. Again, these observations fit well with our aforementioned suggestion that the etiology of stroke is a polygenic multifactorial model caused by a sum of interactions between genetic and environmental factors. In the post-genomics era, it is important to note that the growing list of non-synonymous polymorphisms in the DNA repair genes may provide candidates for further investigations of stroke. Being a heterogeneous group of LAA stroke that reflects a complicated etiology modified by known factors, including hypertension, hyperlipidemia, diabetes mellitus, and obesity ${ }^{45,46)}$, genome-wide association studies with a larger sample size that consider extensive information regarding genetic and environmental factors on modulating LAA stroke risk should contribute to better understanding of the physiology and etiology of LAA stroke development.

\section{Conclusion}

Our hospital-based case-control study used a multigenic model of DNA repair pathway genes to evaluate individual vulnerability to oxidative stress through cigarette smoking and investigated the relationship between individuals who carried polymorphisms of DNA repair genes and the risk incidence in smokers with LAA stroke. To the best of our knowledge, this is the first study of its kind. Based on this epistatic analysis, susceptible polymorphisms for BER and NER pathway genes may address the issue of risk assessment for LAA stroke among ethnic Chinese in Taiwan.

\section{Acknowledgements}

This study was supported by a research grant from the Armed Forces Taoyuan General Hospital, Taoyuan, Taiwan.

\section{Conflicts of Interest}

The authors declare that they have no competing interests.

\section{References}

1) Andreassi MG, Botto N: Genetic instability, DNA damage and atherosclerosis. Cell Cycle, 2003; 2: 224-227

2) Gao X, Yang H, ZhiPing T: Association studies of genetic polymorphism, environmental factors and their interaction in ischemic stroke. Neurosci Lett, 2006; 398: 172177

3) Csiszar A, Podlutsky A, Wolin MS, Losonczy G, Pacher P, Ungvari Z: Oxidative stress and accelerated vascular aging: implications for cigarette smoking. Front Biosci, 2009; 14: 3128-3144

4) White WB: Smoking-related morbidity and mortality in the cardiovascular setting. Prev Cardiol, 2007; 10: 1-4

5) Izzotti A, D’Agostini F, Balansky R, Degan P, Pennisi TM, Steele VE, De Flora S: Exposure of mice to cigarette smoke and/or light causes DNA alterations in heart and aorta. Mutat Res, 2008; 644: 38-42

6) Ambrose JA, Barua RS: The pathophysiology of cigarette smoking and cardiovascular disease: an update. J Am Coll Cardiol, 2004; 43: 1731-1737

7) Calderon-Ezquerro C, Sanchez-Reyes A, Sansores RH, Villalobos-Pietrini R, Amador-Munoz O, Guerrero-Guer- 
ra C, Calderon-Segura ME, Uribe-Hernandez R, GomezArroyo S: Cell proliferation kinetics and genotoxicity in lymphocytes of smokers living in Mexico City. Hum Exp Toxicol, 2007; 26: 715-722

8) Chen HW, Chien ML, Chaung YH, Lii CK, Wang TS: Extracts from cigarette smoke induce DNA damage and cell adhesion molecule expression through different pathways. Chem Biol Interact, 2004; 150: 233-241

9) Henderson SO, Bretsky P, Henderson BE, Stram DO: Risk factors for cardiovascular and cerebrovascular death among African Americans and Hispanics in Los Angeles, California. Acad Emerg Med, 2001; 8: 1163-1172

10) Lee CR, North KE, Bray MS, Avery CL, Mosher M J, Couper DJ, Coresh J, Folsom AR, Boerwinkle E, Heiss G, Zeldin DC: NOS3 polymorphisms, cigarette smoking, and cardiovascular disease risk: the Atherosclerosis Risk in Communities study. Pharmacogenet Genomics, 2006; 16: 891-899

11) Habib SL: Insight into mechanism of oxidative DNA damage in angiomyolipomas from TSC patients. Mol Cancer, 2009; 8: 13

12) Loft S, Svoboda P, Kasai H, Tjonneland A, Vogel U, Moller P, Overvad K, Raaschou-Nielsen O: Prospective study of 8-oxo-7,8-dihydro-2'-deoxyguanosine excretion and the risk of lung cancer. Carcinogenesis, 2006; 27: $1245-1250$

13) Zhang YJ, Weksler BB, Wang L, Schwartz J, Santella RM: Immunohistochemical detection of polycyclic aromatic hydrocarbon-DNA damage in human blood vessels of smokers and non-smokers. Atherosclerosis, 1998; 140: 325-331

14) Wang Y: Bulky DNA lesions induced by reactive oxygen species. Chem Res Toxicol, 2008; 21: 276-281

15) Rossner P Jr, Terry MB, Gammon MD, Zhang FF, Teitelbaum SL, Eng SM, Sagiv SK, Gaudet MM, Neugut AI, Santella RM: OGG1 polymorphisms and breast cancer risk. Cancer Epidemiol Biomarkers Prev, 2006; 15: 811815

16) Chiang FY, Wu CW, Hsiao PJ, Kuo WR, Lee KW, Lin JC, Liao YC, Juo SH: Association between polymorphisms in DNA base excision repair genes XRCC1, APE1, and ADPRT and differentiated thyroid carcinoma. Clin Cancer Res, 2008; 14: 5919-5924

17) Iyer N, Reagan MS, Wu KJ, Canagarajah B, Friedberg EC: Interactions involving the human RNA polymerase II transcription/nucleotide excision repair complex TFI$\mathrm{IH}$, the nucleotide excision repair protein XPG, and Cockayne syndrome group B (CSB) protein. Biochemistry, 1996; 35: 2157-2167

18) Vodicka P, Kumar R, Stetina R, Sanyal S, Souce P, Haufroid V, Dusinska M, et al: Genetic polymorphisms in DNA repair genes and possible links with DNA repair rates, chromosomal aberrations and single-strand breaks in DNA. Carcinogenesis, 2004; 25: 757-763

19) da Silva TA, Fontes FL, Coutinho LG, de Souza FR, de Melo JT, de Souto JT, Leib SL, Agnez-Lima LF: SNPs in DNA repair genes associated to meningitis and host immune response. Mutation Res, 2011: 713: 39-47

20) Tajouri L, Mellick AS, Ashton KJ, Tannenberg AE, Nagra RM, Tourtellotte WW, Griffiths LR: Quantitative and qualitative changes in gene expression patterns characterize the activity of plaques in multiple sclerosis. Brain Res Mol Brain Res, 2003; 119: 170-183

21) Warchol T, Mostowska A, Lianeri M, Lacki JK, Jagodzinski PP: XRCC1 Arg399Gln Gene Polymorphism and the Risk of Systemic Lupus Erythematosus in the Polish Population. DNA Cell Biol, 2011; Epub ahead of print

22) Coppede F, Migheli F, Ceravolo R, Bregant E, Rocchi A, Petrozzi L, Unti E, Lonigro R, Siciliano G, Migliore L: The hOGG1 Ser326Cys polymorphism and Huntington's disease. Toxicology, 2010; 278: 199-203

23) Ding H, Xu Y, Bao X, Wang X, Cui G, Wang W, Hui R, Wang DW: Confirmation of genomewide association signals in Chinese Han population reveals risk loci for ischemic stroke. Stroke, 2009; 41: 177-180

24) Ikram MA, Seshadri S, Bis JC, Fornage, M, DeStefano, A L, Aulchenko YS, Debette S, et al: Genomewide association studies of stroke. N Engl J Med, 2009; 360: 17181728

25) Yip PK, Jeng JS, Lee TK, Chang YC, Huang ZS, Ng SK, Chen RC: Subtypes of ischemic stroke. A hospital-based stroke registry in Taiwan (SCAN-IV). Stroke, 1997; 28: 2507-2512

26) Adams HP, Jr., Bendixen BH, Kappelle LJ, Biller J, Love BB, Gordon DL, Marsh EE 3rd: Classification of subtype of acute ischemic stroke. Definitions for use in a multicenter clinical trial. TOAST. Trial of Org 10172 in Acute Stroke Treatment. Stroke, 1993; 24: 35-41

27) Kumar R, Hoglund L, Zhao C, Forsti A, Snellman E, Hemminki K: Single nucleotide polymorphisms in the XPG gene: determination of role in DNA repair and breast cancer risk. Int J Cancer, 2003; 103: 671-675

28) Sreeja L, Syamala VS, Syamala V, Hariharan S, Raveendran PB, Vijayalekshmi RV, Madhavan J, Ankathil R: Prognostic importance of DNA repair gene polymorphisms of XRCC1 Arg399Gln and XPD Lys751Gln in lung cancer patients from India. J Cancer Res Clin Oncol, 2008; 134: 645-652

29) Peterson HB, Kleinbaum DG: Interpreting the literature in obstetrics and gynecology: II. Logistic regression and related issues. Obstet Gynecol, 1991; 78: 717-720

30) Curtis D, Gurling HM: Using a dummy quantitative variable to deal with multiple affection categories in genetic linkage analysis. Ann Hum Genet, 1991; 55: 321-327

31) Ananth CV, Kleinbaum DG: Regression models for ordinal responses: a review of methods and applications. Int J Epidemiol, 1997; 26: 1323-1333

32) Shen J, Deininger P, Hunt JD, Zhao H: 8-Hydroxy-2'-deoxyguanosine (8-OH-dG) as a potential survival biomarker in patients with nonsmall-cell lung cancer. Cancer, 2007; 109: 574-580

33) Coppede F, Mancuso M, Lo Gerfo A, Carlesi C, Piazza S, Rocchi A, Petrozzi L, Nesti C, Micheli D, Bacci A, Migliore L, Murri L, Siciliano G: Association of the hOGG1 Ser326Cys polymorphism with sporadic amyotrophic lateral sclerosis. Neurosci Lett, 2007; 420: 163-168

34) Daimon M, Oizumi T, Toriyama S, Karasawa S, Jimbu Y, Wada K, Kameda W, Susa S, Muramatsu M, Kubota I, Kawata S, Kato T: Association of the Ser326Cys polymorphism in the OGG1 gene with type 2 DM. Biochem Bio- 
phys Res Commun, 2009; 386: 26-29

35) Li H, Hao X, Zhang W, Wei Q, Chen K: The hOGG1 Ser326Cys polymorphism and lung cancer risk: a metaanalysis. Cancer Epidemiol Biomarkers Prev, 2008; 17: 1739-1745

36) Wang CL, Lin TH, Lin HY, Sheu SH, Yu ML, Hsiao PJ, Lin KD, Hsu C, Yang YH, Shin SJ: The 8-oxoguanine glycosylase I (hOGG1) Ser326Cys variant affects the susceptibility to multi-vessel disease in Taiwan coronary artery disease patients. Thromb Res, 2010; 126: 319-323

37) Zhang J, Gu SY, Zhang P, Jia Z, Chang JH: ERCC2 Lys751Gln polymorphism is associated with lung cancer among Caucasians. Eur J Cancer, 2010; 46: 2479-2484

38) Shen J, Desai M, Agrawal M, Kennedy DO, Senie RT, Santella RM, Terry MB: Polymorphisms in nucleotide excision repair genes and DNA repair capacity phenotype in sisters discordant for breast cancer. Cancer Epidemiol Biomarkers Prev, 2006; 15: 1614-1619

39) Gupta RC, Arif JM, Gairola CG: Enhancement of pre-existing DNA adducts in rodents exposed to cigarette smoke. Mutat Res, 1999; 424: 195-205

40) Izzotti A, Camoirano A, Cartiglia C, Tampa E, De Flora S: Formation of DNA adducts in the aorta of smoke-exposed rats, and modulation by chemopreventive agents. Mutat Res, 2001; 494: 97-106

41) Oberg M, Jaakkola MS, Woodward A, Peruga A, Pruss-
Ustun A: Worldwide burden of disease from exposure to second-hand smoke: a retrospective analysis of data from 192 countries. Lancet, 2011; 377: 139-146

42) Woodward M, Lam TH, Barzi F, Patel A, Gu D, Rodgers A, Suh I; Asia Pacific Cohort Studies Collaboration: Smoking, quitting, and the risk of cardiovascular disease among women and men in the Asia-Pacific region. Int J Epidemiol, 2005; 34: 1036-1045

43) Harms C, Salama SA, Sierra-Torres CH, Cajas-Salazar N, Au WW: Polymorphisms in DNA repair genes, chromosome aberrations, and lung cancer. Environ Mol Mutagen, 2004; 44: 74-82

44) Rzeszowska-Wolny J, Polanska J, Pietrowska M, Palyvoda O, Jaworska J, Butkiewicz D, Hancock R: Influence of polymorphisms in DNA repair genes XPD, XRCC1 and MGMT on DNA damage induced by gamma radiation and its repair in lymphocytes in vitro. Radiat Res, 2005; 164: $132-140$

45) Deleu D, Hamad AA, Kamram S, El Siddig A, Al Hail H, Hamdy SM: Ethnic variations in risk factor profile, pattern and recurrence of non-cardioembolic ischemic stroke. Arch Med Res, 2006; 37: 655-662

46) Park JH, Kwon HM: Association between metabolic syndrome and previous ischemic lesions in patients with intracranial atherosclerotic stroke. Clin Neurol Neurosurg, 2008; 110: 215-221 